\title{
HIPPOCAMPAL MALFORMATION AND FEBRILE SEIZURES
}

The MRIs of 13 members of two families with febrile convulsions (FC) and 10 members without FC were compared with 23 control subjects in a study at the Universities of Magdeburg and Bonn, Germany. One member of each family with febrile convulsions had developed temporal lobe epilepsy (TLE) and both TLE patients showed left-sided hippocampal sclerosis. All subjects with febrile convulsions and without subsequent epilepsy and six unaffected relatives showed asymmetric hippocampal volumes, the left smaller than right. The pattern and left-sided involvement of hippocampal sclerosis in TLE patients was similar to that in their relatives without epilepsy. Additional subcortical heterotopias were found in one FC patient. (Fernandez G, Effenberger O, Vinz B et al. Hippocampal malformation as a cause of familial febrile convulsions and subsequent hippocampal sclerosis. Neurology April 1998;50:909-917). (Reprints: Dr Guillen Fernandez, Klinik fur Epileptologie, Sigmund-Freud-Str, 25, 53105 Bonn, FR Germany).

COMMENT. Patients with familial febrile convulsions may have a subtle, inherited form of pre-existing hippocampal malformation that predisposes to and is not a consequence of febrile convulsions. The hippocampal malformation may facilitate the development of hippocampal sclerosis in patients with temporal lobe epilepsy and antecedent febrile convulsions. A dominant mode of inheritance is suggested. These findings are interesting and provocative but not entirely supported by previous reports.

In an editorial, Sloviter RS and Pedley TA comment that other large series of surgical resections for TLE had not found a predilection for the left side in patients with hippocampal sclerosis (Neurology April 1998;50:846-849). Bilateral hippocampal abnormalities and dual pathologies, focal cortical dysplasia occurring with mesial temporal sclerosis, were reported in patients with TLE treated at the University of Alabama (see Ped Neur Briefs April 1998;12:26). Routine MRIs may miss subtle abnormalities, and special techniques are necessary to uncover hippocampal structural anomalies.

\section{RISK OF FEBRILE SEIZURE RECURRENCE AND OUTCOME}

The influence of febrile episodes, a risk factor for recurrence of febrile seizures, and outcome in 180 children followed prospectively after their first febrile seizure, were studied at the University of Oulu, Finland. In a 2-year followup of 156 patients, each febrile episode increased the risk of febrile seizure recurrence by $18 \%$. Of 38 patients with recurrences, 27 had single and 11 had multiple recurrences. EEG spikes or spike-and-wave abnormalities occurred in $14 \%$ of 35 children with recurrences and in $9 \%$ of 121 without recurrences. Each degree of temperature (Celsius) increase during subsequent infections almost doubled the risk of seizure recurrence. Age, sex, or family history of febrile seizures or epilepsy were not risk factors for febrile seizure recurrence. (Tarkka R, Rantala H, Uhari M, Pokka T. Risk of recurrence and outcome after the first febrile seizure. Pediatr Neurol 1998;18:218-220). (Respond: Dr Heikki Rantala, Department of Pediatrics, University of Oulu, FIN-90220 Oulu, Finland).

COMMENT. This study corroborates previous reports that the height of a fever is the most important determinant of a febrile convulsion, and treatment aimed at prevention of infection and a rise in temperature to the "threshold convulsive level" should decrease the risk of febrile seizure recurrence (Millichap JG. Febrile Convulsions. New York, Macmillan, 1968). Rantala and colleagues show that the frequency of febrile episodes after the initial seizure is a 
risk factor for recurrence of febrile seizures. Children with recurrences have higher temperatures than those without, supporting the theory of a febrile seizure threshold dependent on the height of the temperature.

Prediction of febrile seizures in siblings was studied prospectively in 129 children with FC at Sophia Children's Hospital, Rotterdam, The Netherlands (van Esch A, Steyerberg EW, van Duijn CM et al. Eur I Pediatr April 1998;157:340$344)$. The risk of FS in siblings was $10 \%$, more than twice the population risk (4\%). The overall risk of FS in first degree relatives of FS probands was $7 \%$. The risk was increased to $16 \%$ in siblings with recurrent FS and $25 \%$ in siblings with an affected parent. A polygenic mode of inheritance seemed likely. A prediction model based on three risk factors was developed. The risk factors are: 1) FS in the parents; 2) age at first FS of proband under 1 year; and 3) FS recurrence in the proband. The risk of FS in siblings of FS probands may increase to $46 \%$ with 2 or 3 risk factors present and it falls to less than $10 \%$ if a sibling is unaffected up to 3 years of age.

\section{CHILDHOOD EPILEPSY TREATMENT STRATEGIES}

Treatment strategies employed in 494 children with various seizure types and remission frequencies were studied prospectively at multiple hospital centers in the Netherlands. In $142(29 \%)$ treatment was initially withheld, and after 2 years $17 \%$ were still untreated, none suffering serious complications. Of 416 treated with AEDs, $88 \%$ received valproic acid or carbamazepine initially, and $40 \%$ did not respond successfully. Reasons for treatment failures included recurrent seizures $(28 \%)$, and intolerable side effects $(11 \%)$. Rashes occurred in $15(4 \%), 14$ with carbamazepine ( $10 \%$ of all children who received carbamazepine). The chance of achieving remission was negatively associated with the number of AED regimens tried. Alternative AEDs included phenytoin, phenobarbital, ethosuximide, and vigabatrin. If 3 regimens had failed, the chance of remission with alternative therapy was only $10 \%$. The epilepsy was considered intractable in only $7 \%$. A distinction was made between acceptable control, with low seizure frequency or severity, and intractable epilepsy. (Carpay HA, Arts WFM, Geerts AT et al. Epilepsy in childhood. An audit of clinical practice. Arch Neurol May 1998;55:668-673). (Reprints: Willem FM Arts MD, PhD, Department of Child Neurology, University Hospital-Sophia Children's Hospital, Dr Molewaterplein 60, 3015 GJ Rotterdam, The Netherlands).

COMMENT. The initial choice of AED therapy, mainly valproic acid or carbamazepine, fails to control childhood epilepsies in $40 \%$ of cases. Alternative therapies are necessary because of seizure recurrences or side effects, especially skin rash, a frequent complication of carbamazepine treatment. Newer AEDs such as gabapentin (Neurontin ${ }^{\circledR}$ ), with a relatively low incidence of side effects, should increase the remission frequency of childhood epilepsies, especially partial seizures. In one large study of gabapentin as add-on therapy of 705 adult patients, skin rash was reported in only $0.5 \%$ compared to a $10 \%$ incidence with carbamazepine (Progress in Pediatric Neurology III, 1997; pp122-125).

\section{BILATERAL RASMUSSEN CHRONIC ENCEPHALITIS}

Two Peruvian brothers, ages 16 months and 5 years, with alternating epilepsia partialis continua are reported from the Montreal Neurological Hospital, McGill University, Canada, and Loyola University, Chicago. Seizures began at 6 months in one child and at 4 months in the other, both associated with a febrile illness. Severe psychomotor regression and cerebral atrophy developed rapidly. A brain biopsy in one child revealed chronic encephalitis with changes compatible 